International Journal of English Literature and Social Sciences
Vol-6, Issue-1; Jan-Feb, 2021

\title{
Frequency and Factors of Non-Reporting of Violence against Women in Tabuk City
}

\author{
Mary Grace Baddongen Malawis-Ignacio, Ph.D. \\ College of Criminal Justice Education, Kalinga State University, Philippines
}

Received: 14 Nov 2020; Received in revised form: 21 Jan 2021; Accepted: 10 Feb 2021; Available online: 25 Feb 2021

(C)2021 The Author(s). Published by Infogain Publication. This is an open access article under the CC BY license

(https://creativecommons.org/licenses/by/4.0/).

\begin{abstract}
Purpose: Violence against women is still a pervasive problem worldwide. In the City of Tabuk, Kalinga, Philippines, violence against women is undoubtedly existing. This study aimed to determine the existence of unreported violence against women (VAW) committed by intimate partners of women-residents in Tabuk City that are not reported. It also determined why these cases are not reported to proper authorities. Methods: The data were drawn thru informal interviews involving 356 randomly selected women-residents from the different urban barangays of Tabuk City, Kalinga. Women-residents included as respondents were in an intimate relationship during or before the study's conduct. Results: The data gathered showed that psychological violence is the most (64.89\%) pervasive form of VAW experienced by respondents. The data also revealed that all forms of VAW exist in the study's locale to a varying frequency. This experience's nonreporting is primarily due to the victim's hope that the case will be settled in the family and need not be publicized. Her fear that her children will emotionally and physically suffer are also among the top factors why violence cases were not reported to the authority. Conclusion: Women in an intimate relationship experienced one or more modes of violence during or after their relationship duration. However, the frequency and reason for not reporting may vary from one victim-survivor to another. A need to empower victim-survivors that they may not hesitate to report violence committed against them by their intimate partners may be initiated. For this reason, an extension project with the banner program of "Protection of Women against Violence" was initiated and extended to the different barangays starting from two of the barangays where women respondents come.
\end{abstract}

Keywords - Violence against women (VAW); victim-survivor; frequency of violence; intimate relationship, factors of non-reporting VAW; psychological violence.

\section{INTRODUCTION}

Violence against Women (VAW) refers to any act of gender-based violence that results or is likely to result in physical, sexual, or mental harm or suffering to women, including threats of such acts, coercion or arbitrary deprivation of liberty, whether occurring in public or private life" (United Nation (UN) Declaration on the Elimination of Violence against Women). This term includes physical abuse, sexual abuse, psychological violence, non-spousal violence, and sexual harassment and intimidation at work, educational institutions, and elsewhere. As herein used, violence is defined as the use of physical force to cause injury or abuse.
In the Philippines, violence against women (VAW) appears as one of the pervasive social problems. According to the 2008 National Demographic and Health Survey conducted by the National Statistics Office, one in five Filipino women age 15-49 has experienced physical violence since age 15. It is indeed alarming that despite efforts to address the concern, VAW persists.

Lack of concrete information to show the extent of VAW in the country as many cases of violence against women are unreported due to women victims' "culture of silence." Many of the victims are ashamed to relate their experiences. In contrast, others tend to dismiss their ordeal 
due to their lack of faith in the country's justice system caused by

Assaults to wives by their partners may have been declared illegal, but reports show that it still exists. It continues to be practiced by not only husbands but also by boyfriends or partners of women. No matter how slight the abuse or violence, it is still abuse or violence. This act should not be condoned.

The information gathered from informal interviews and observation of the researcher with some community members reveals many VAW cases that even surpassed the PNP record. The presence of unreported VAW cases, and why these are not reported drives the researcher to conduct this study.

In the city of Tabuk, a pre-survey conducted by the researcher in some of the urban barangays reveals that there are VAW cases which, when compared to the number of reported cases provided by Tabuk City Police Office, is higher, especially when we consider the fact that the presurvey was conducted only in some urban barangays out of the 42 barangays of Tabuk City. The pre-survey also revealed that most of the barangays do not have an established VAW Desk. Women who experience physical, psychological, and sexual abuse can seek assistance and flee from the violence inflicted on them. This alarming information gathered motivated the researcher to conduct the study to verify the pre-survey and barangay officials' capability to address VAW cases.

This study may serve as a basis for the local government unit of Tabuk City, even Kalinga province, in identifying factors that hinder the reporting of VAW cases. This study may encourage the local government to initiate programs that will increase the residents' awareness of what may be done and what will be given to VAW victimssurvivors, which may further assure and guarantee protection against violence. It may also serve as a reminder for the strict implementation of keeping records of VAW cases reported regardless of whether it was eventually settled or retracted by the complainant/victim-survivor. Further, this may also prompt advocates to form a group or an organization that will tailor the needs or services to protect women experiencing violence. The advocacy group may help ensure the highest quality of assistance and protection to VAW victims-survivors and facilitate the provision of services to VAW V-S and their families. This study may serve as a basis for the Higher Educational Institutions in the conceptualization of extension activity geared towards the empowerment of barangay officials and the community to address issues and concerns relative to women's protection.

\section{CONCEPTUAL FRAMEWORK}

\subsection{Frequency of Violence}

Social Disorganization Theory developed by the Chicago School of Criminology directly links crime to neighborhood ecological characteristics. It suggests that a person's residential location is more significant than the person's characteristics when predicting criminal activity and that criminality is more likely to appear in a disadvantaged urban neighborhood. The neighborhood itself determines the probability of the existence of violence according to the aforementioned theory. Disorganized areas identified by their relatively high level of change, fear, instability, incivility, poverty, and deterioration directly influence the area's delinquency rate. Shifting from the connection of location and violence is the explanation of the cycle of violence. The cycle of violence of theory has another way of explaining the existence of violence.

This cycle of violence theory states that domestic violence is characterized by three distinct phases repeated repeatedly in the abusive relationship. As a result, domestic abuse rarely involves a single isolated incident of violence. Instead, the abuse becomes a repetitive pattern in the relationship (Crossman, 2017).

Closely related to Cycle of Violence Theory is the family conflict model that assumes that the victim's actions somehow justify the batterer's abusive response. Even when women use violence in an intimate relationship, they generally do so to defend themselves from an assault. According to this description, batterers follow a "cycle of violence" with intermittent violent and repentant episodes. However, advocates in the United States found that this description of the violence was not consistent with women's experiences. Many women reported that their partners never repented in their violent relationships and that violence was not cyclical but rather a constant presence in their lives (Minnesota Advocates for Human Rights, 2003). Family conflict theory gives justification on why violence exists in an intimate relationship. It is this theory that blames women. This theory tells that women's actions trigger men to inflict violence, which is the same when saying that the violence should not have existed if not for the actions or words uttered by women.

Another explanation of why violence is committed is somewhat similar to family conflict theory. According to 
the "loss of control" theory, violence exists when a man is drunk, where he loses control of himself, and when he loses the ability to control his anger or frustration (Minnesota Advocates for Human Rights, 2003). This theory states that violence inflicted on women by their partners is committed not because the man wants to hurt the woman but because he loses the ability to control himself. Both theory's explanation implies that the violence is a reaction of the man to their women-partners' actions or words.

Large cities are said to be characterized by anonymity. In a world of strangers, men lose their feelings of responsibility for their fellow man. The sheer number of people in the large city ensures that people with criminal tendencies can find people with similar tendencies to provide social support for their criminality (Braithwaite, n.d.). This idea implies that when people in the community do inflict violence against their partners, the possibility of committing violence by those who see other people doing it is greater. This focus on the characteristics of people residing within an area is somewhat similar to what Rawson and Nolan claimed. Rawson W. Rawson states that crime statistics suggest a link between population density and crime rates (Mazuma Capital Management, 2017).

\subsection{Factors Contributing to Non-reporting of Violence against Women}

On the part of the victim, non-reporting may be explained by the Learned Helplessness Theory. This theory claimed that women suffered from a "learned helplessness" due to repeated battering that prevented them from resisting the violence or leaving the relationship. However, the "learned helplessness" theory did not account for the fact that there are many social, economic, and cultural reasons a woman might choose to stay in an abusive relationship. They may fear retaliation against themselves or their children, or they may not support themselves or their children financially. Their family and community may ostracize them if they leave. This theory is also inconsistent because women surviving in abusive relationships attempt to leave often and routinely act in very conscious ways to minimize the abuse directed at them and protect their children (Minnesota Advocates for Human Rights, 2003).

Non-reporting of violence on the part of those who witness or have knowledge about such can be explained by the psychological phenomenon in which people are less likely to take action when in the presence of a large group of people called diffusion of responsibility. This is often used to explain the bystander effect, which suggests that the greater the number of people present, the less likely people will help a person in distress. This is not to suggest that people are not acting because they lack compassion. However, they may not process a traumatic situation as it unfolds, especially when others are around (Cherry, 2017).

\section{METHODS}

\subsection{Study Design}

This study made use of quantitative and qualitative approaches, specifically the descriptive method. Frequency of unreported violence against women and the factors of not reporting was gathered using informal interview using a semi-structured interview guide. The respondents were 356 women-residents from 13 different urban barangays of Tabuk City. As used in the study, women-residents are those women with intimate relationships during or before the study's conduct.

This study's locale Tabuk City is the capital of Kalinga Province, Cordillera Administrative Region, Philippines, and comprises 42 barangays. However, this study was delimited to the urban barangays.

Women-residents who experienced violence provided their reasons for not reporting. In contrast, those who did not experience violence provided their perception on why those that experienced violence did not report it to the proper authorities.

Confidentiality of information collected from respondents is of fundamental importance. In this connection, the interview was conducted only in a private setting. The participant was informed that if she desires, rescheduling or relocation of the interview to a time or place that may be safer or more convenient.

\subsection{Data Analysis}

The data gathered was treated using descriptive and inferential statistical tools.

The percentage was used to describe the factors contributing to the non-reporting of violence against women.

The respondents' perception of the factors affecting the non-reporting of violence committed against women in Tabuk City was interpreted based on the ranks given by the different women-residents of the respondent-barangays.

Pearson's Product-Moment Correlation Coefficient used a linear correlation in determining the relationship between the frequency of violence and the urban barangay population in Tabuk City. 


\section{FINDINGS}

\subsection{Frequency of the Commission of Violence against Women in the Urban Barangays of Tabuk City}

Violence against women in this part was focused on those committed by the intimate partners of the women-residents from the urban barangays of Tabuk City. Data from this was gathered from 356 women-residents of the urban barangays of Tabuk City.

The total number of unreported VAW incidents from the 13 urban barangays of Tabuk City is 225. This data means that $1.12 \%$ of all the women-residents from the 13 urban barangays experienced VAW but chose not to report it to the proper authorities. This result implies that VAW within the urban barangays of Tabuk City exists. This further implies that the recorded VAW incidents are low, considering that the data gathered was from the 13 barangays only. The low rate of VAW incidents in Tabuk City does not support Social Disorganization Theory, directly linking the crime to neighborhood ecological characteristics. Though the study focuses on disorganized areas because of mixed cultures, relative deprivation, and poverty, the rate of VAW is still not as high as what is expected to be present in disorganized areas. Urban barangays in the City of Tabuk exhibit relative deprivation because residential and commercial buildings, affluent and low-income families, live close. Being urban, most of the residents are of different cultures, from different places, and with different religious sects. Poverty is also still visible in these areas since households with income below the poverty threshold and households with income below the food threshold are still high. Poverty is also shown by the existence of unemployed members of the labor force (CBMS Census 2015-2016).

There were $.66 \%$ women-residents at Barangay 12 who have acknowledged having experienced psychological violence. This data is equivalent to $23.97 \%$ of the overall number of women-residents from the different urban barangays of Tabuk City who experienced the same form of violence. Most of the women who acknowledged having experienced psychological violence experienced physical and or sexual violence at the same time.

Psychological violence is the Form of VAW experienced by $64.9 \%$ of the 225 women-residents who acknowledged having experienced it. It may not be readily noticeable but brought emotional suffering to these women. This data implies that there are women who appear to be okay from the outside but crying inside. These are those who deal with their emotional suffering thru psychological projection. They smile instead of admitting the existence of the pain. These are those who believe that these sufferings are ordinary in a relationship. These are those who believe that they may be at fault. These are those who believe that if God had allowed them to be united through matrimony, then experiencing such abuse is part of it or normal. They stick to the understanding that "what therefore God hath joined together, let no one separate" (Mark 10: 9, NIV). Thus, even when they are suffering emotionally, some bear it. This passage implies that women's choice of being strong outward is their way of protecting themselves and their family from being broken.

The total number of physical violence is similar to the number of reported VAW cases in the City of Tabuk as per the Tabuk City Police Office's record. This shows that the record does not reflect the actual prevalence of VAW in Tabuk City. This implies there are still victim-survivors who need protection yet are not given the proper attention. These do not come to the attention of proper agencies in charge of addressing VAW victim-survivors.

Most (.74\%) of the physical violence committed as acknowledged by the women-residents who experienced it was in Barangay 7. This is equivalent to $17.54 \%$ of the overall number of women-residents from the 13 barangays who acknowledged having experienced the same form of violence. This figure negates Rawson, who suggests a link between population density and crime rates. Barangay 7 has a population $(1,352)$ lower than Barangay $2(5,295)$ but has a higher VAW incidence. This result also does not support Nolan III (2004), who also claimed that the crime rate and population are closely related.

A total of $.17 \%$ of women-residents of barangay 6 acknowledged having experienced sexual violence. This means that $25 \%$ of women-residents from the 13 urban barangays who acknowledged experiencing sexual violence came from Barangay 6 . Thus, .015\% of women residents of the urban barangays of Tabuk experienced sexual violence.

The type of violence which is least (4.44\%) experienced by the women-residents in the urban barangays of Tabuk City was economic violence. This amounts to $0.05 \%$ of the total women-residents of the urban barangays of Tabuk City. This implies that the women-residents are not financially dependent on their partners. This is supported by their common statement that they can also have money of their own without asking or expecting it from their partners.

This study's findings are similar to the result of the study conducted by the group of Chiashvili, which reveals that a relatively small number of women in Georgia 
acknowledged being victims of physical or sexual violence. Women who have experienced sexual violence also acknowledged having suffered from psychological violence ("Examination of Fourth," 2014). Furthermore, Antai, Braithwaith, Oke, and Lopez (2014) mentioned in their study "The Effect of Economic, Physical, and Psychological Abuse on Mental Health: A Population-Based Study of Women in the Philippines," physical abuse in intimate relationships is often accompanied by psychological abuse and in one-third to over one-half of cases by sexual abuse. It was also mentioned that psychological abuse might have equally deleterious effects on women's mental health, if not more so, than physical abuse (Alfonso, Linares, Navarro, Ros, Echeburua, \& Martinez, 2006).

Psychological violence, although more subtle, is an insidious form of violence. The intangible nature of psychological abuse makes it harder to define and report, leaving the woman in a situation where she is often made to feel mentally destabilized and powerless (Domestic Violence against Women and Girls, 2000). This is also similar to what was mentioned by Patricia Evans as cited by Brogaard (n.d.) in her article 15 Common Forms of Verbal Abuse in Relationships that there are several categories of verbal abuse that are either subtle or obvious. According to Brogaard, subtle or obvious ways of verbal abuse can be thought of as ways in which someone tries to dominate or control their partner.

The highest number (41) of VAW incidents was recorded in Barangay 2. This means that $18.22 \%$ of all VAW incidents acknowledged by the women-residents from the 13 urban barangays who participated in this study came from Barangay 2. This further means that $.20 \%$ of womenresidents from the respondent barangays experienced VAW from their partners. Barangay 2 is also the barangay that has the highest number of women-residents. Barangay 2 is also one of the barangays with a heterogeneous population in terms of tribes. This implies that the higher the population, the higher VAW incidents. Further, the more heterogeneous the population, the more possibility of the existence of VAW.

This supports the view of the Concentric Zone Theory on Crime, arguing that crime increases toward the inner city where the population is bigger because of immigration, industrialization, and urbanization ("Theories of Crime", n.d.). These characteristics are present on the different urban barangays of study as they are usually the areas where people from the neighboring municipalities stay to study or do business. This turnover rate in population or population instability and people's heterogeneity from different cultural backgrounds (ethnic diversity) causes social disorganization. Further, crime was a dependent variable, i.e., it depended on all the other factors that might give rise to crime (Nolan III, 2004), such as the factors mentioned above.

In contrast, there was one barangay that is almost homogenous in terms of culture. Most of the residents are of the same tribal affiliation. This is one of the significant reasons why according to the barangay officials interviewed (personal communication, January 19, 2017), even if there are cases of violence committed against women by their intimate partners in their barangay, a settlement is done through their settlement tradition where elders intervened. This elders' intervention usually results in the settlement and non-reporting of such violence to the proper agencies. Cases that are not settled are referred to the city social welfare office. However, according to the barangay official, almost all violent incidents against women are settled without being reported and recorded in the barangay. This is regardless of whether the barangay officials participated in the settlement. According to the barangay official interviewed (personal communication, January 19, 2017), there was even this case of domestic violence, physical injury, and rape that was settled through bodong as this is allowed in their indigenous justice. Article VIII, Section 2, of the Pagta, the penalty for rape is 12 carabaos. This supports collective efficacy theory claiming that when most community or neighborhood members are acquainted and on good terms with one another, the greater the community's capacity for supervision because acquaintances are willing to intervene when one behaves unacceptably (Higgins \& Hunt, 2016).

In another barangay, the barangay chairman had narrated that the cases of violence are not recorded, but these are settled. This implies that women-residents chose settlement as an option in addressing the violence. This, however, is against the law prohibiting the settlement of VAW cases.

According to the barangay chairman (personal communication, January 19, 2017), the process he implements in addressing intimate partner violence is: when there is a complaint, he will send a barangay tanod to summon the perpetrator; he will then counsel and warn the perpetrator that when it is repeated, he will recommend the removal of their names from among the beneficiary of 4Ps (if the involved is a beneficiary of 4Ps). For those who are not members of 4Ps, the barangay chairman said that once summoned, he warns them that if it is repeated, then he will be the one to report and accompany the police officers in apprehending them personally. So far, according to the 
barangay official, this method was effective in preventing the re-occurrence of violence.

The aforementioned reasons might be one reason why, even if there were only 59 cases of Violence against Women in Tabuk, violence against women reported by the women-residents when interviewed is higher. Through their indigenous justice system, settlement played a significant role in the non-reporting and recording of violence against women cases in some barangays in Tabuk City.

The result of the study showing the highest number of VAW incidents in a barangay that does not have the highest population does is in contrast with Nolan III (2004), who, thru his research, established a significant positive relationship between crime rate and population size, indicating that the higher populated cities reported the higher crime rates.

The occurrence of violence is most of the time linked with misunderstanding. Misunderstanding is expected in an intimate relationship. However, abusive behavior is never acceptable; thus, misunderstanding in an intimate relationship is not enough reason to resort to abusive behavior (Smith and Segal, 2015). The implication when violence is resorted to because of misunderstanding is that the couple did not talk about the issue. This further implies that when there is no enough communication between couples, a simple misunderstanding may escalate to violence.

However, the information from the respondents showed that prior the physical or sexual violence is psychological violence, which is usually in the form of uttering insulting words. This, according to Cycle of Violence and Escalation Theory, is how violence increases in severity.

The existence of unreported violence against women in the urban barangays of Tabuk City shows that the recorded VAW cases do not represent the actual figure of Violence against Women cases. Further, the type of violence experienced is also varied. Women-residents consider this to be normal in a relationship. Even the harsh remarks from their partners were considered to be normal in a relationship by most of the women residents.

The data from the women residents relative to the commission of violence also shows that these are also usually committed when their husband or intimate partner is under the influence of alcohol or is having an illicit affair. Thus, the two are identified to be the most common reasons why violence is committed as far as the data from this study is concerned. This is followed by a misunderstanding between the couple, especially for psychological violence. It is during misunderstanding where couples, because of emotional outbursts, utter words that they do not really intend to say. This might be true not only for women who are accordingly more verbal than men but also for men. Thus, insulting words uttered during altercations may not be considered by the woman as a mode of abuse but as normal.

\subsection{Factors Contributing to the Non-Reporting of Violence}

This presents the identified and perceived factors of non-reporting of violence experienced by the womenresidents from the respondent barangays of Tabuk City who may or may not experience violence from their intimate partner. Out of 356 women-residents, 131 did not experience violence; thus, they gave their perceptions as to why women chose not to report their experience.

Most (19.10\%) of the women who responded to the study believe that non-reporting of violence among women is because they hope that the case will be settled between them and that there is no need to bringing it to the public. There are also $32(8.99 \%)$ whose hope of settling the problem is anchored on the intervention of family members or relatives, or elders. This implies that women experiencing violence do not report because of the hope that the problem will be settled without bringing it to the public.

Some $(12.92 \%)$ did not or believed to have not reported the incident of violence because they believe that it is normal in a relationship. This implies that the nonreporting is because the violence is not perceived to be a violation of their rights but is a normal thing in a relationship.

Forgiveness is given by the victim to their husband, or intimate partner is also a factor acknowledged by 56 (15.73\%) women-residents to be one of the causes of nonreporting. This implies that the women who were experiencing violence choose to patch things up instead of reporting it.

Another factor of non-reporting was fear. There were $64(17.98 \%)$ claiming that they did not report to authorities despite experiencing one or more types of violence because they are afraid that their children will emotionally and/or physically suffer. Fifty-seven (15.73\%) attributes it to their fear of having financial struggle if separated from the suspect; $12(3.37 \%)$ said that non-reporting is because of fear of filing a case against the suspect, and seven (1.97\%) attributes it to the fear that the case will not be seriously acted upon by the proper authorities.

The first two leading factors, as identified by 
respondents, both imply high value given to the family that, despite experiencing violence, a woman usually chose to stay because of "family." This somehow supports Durvasula, a psychology professor at California State University, Los Angeles. In an interview, she said, "many times a victim becomes so dependent on her partner for everything that she can't even entertain a reality without him in it" (Kantor, 2014). This might also be attributed to the fact that patriarchal family structure is still dominant in the Philippines. Women who grew up in a patriarchal family structure might have acknowledged that a man has the power and that a woman should follow what men say or just exist to support and obey men.

It is also noteworthy that only $16.01 \%$ of womenresidents identified fear of having financial struggle when separated from their partner as a factor of non-reporting. This is because it is contrary to what several experts in domestic violence that economic reliance is a leading predictor of whether a woman leaves her abuser (Kantor, 2014). Despite the fact that the majority of womenrespondents were housewives who imply being financially dependent on their husbands, they are not afraid to suffer financially but are more concerned about the impact of them leaving their partners to their children.

There are also $3.93 \%$ women-residents also who identified being ashamed of relating their experience as a factor of non-reporting of violence against women. This implies that some women chose to suffer because they believed that people would talk about them when they report it. This further means that some women chose to stay in an abusive relationship because they thought that it is shameful when other people get to know of the abuse. One womanresident, when asked what she thinks is the reason why the woman she knows did not report that she is being abused by her husband, said:

"Aye, ket mabain kano isuna ya. Isu nga uray pukkaw pukkawan ni lakay na ti nagmamadi nga sao wenno danugen na tunggal nabartek adi ket han na latta nga irep report." (She is ashame, that's why even if her husband insults her or punches her when he is drunk she tolerates it).

This reason for non-reporting implies that the Filipino value of shame is so much in place in Tabuk City. This supports the blog on the website of La Solidaridad that the Filipino value of "hiya" regulates how people behave. In this context, the victim-survivor of violence does not report the
VAW incident in order to avoid the feeling of being exposed and unprotected, which may result in being humiliated by the public (Reven, 2010).

The existence of unreported VAW cases support the report

of Felson and Paré (2005) where they cited some researchers who implied that domestic and sexual assaults against women are particularly likely to go unreported. It was also written by them that women's reluctance to report assaults by their male partners had been attributed to fear of reprisal, economic and psychological dependence, and anticipation that the police do not take these charges seriously. In addition, women's reluctance to report sexual assaults has sometimes been attributed to their lack of confidence in a criminal justice system that assigns blame to them rather than to offenders. Further, "relational distance" between the victim and offender is also an issue in the non-reporting of violence. The closer the relational distance between adversaries, the less likely it is to be reported.

Although not included as an indicator under factors contributing to non-reporting of violence by victimsurvivors, one possible reason might be the existence of patriarchal family structure in the different urban barangays.

Another possible factor could be based on a psychological phenomenon in which people are less likely to take action when in the presence of a large group of people called diffusion of responsibility. This is often used to explain the bystander effect, which suggests that the greater the number of people present, the less likely people are to help a person in distress. This is not to suggest that people are not acting because they lack compassion, but they may not be able to process a traumatic situation as it unfolds, especially when others are around (Cherry, 2017). This might be one of the reasons why, even if the neighbors are aware of the existence of violence, they do not report nor intervene as they might have thought that it is not serious enough considering that the victim-survivor is tolerating it. It might be because they thought that their other neighbor might have already called for help.

Any form of violence is never acceptable, be it by a man or a woman, child or adult. Everybody deserves to be free from any form of pain. This is especially true within the family. Comfort, love, and care should be the seeds that grew and nurtured within the home. Thus domestic violence should never be condoned.

Violence against women in Tabuk City is existing, but their culture of resolving conflicts is very much alive. Most 
women rely on this culture of resolving conflicts aside from the different laws protecting women from violence. Nonreporting of violence is somehow connected with the awareness of the laws and the services to be provided to victim-survivors. However, this study shows no connection to such. The law and the services may be in place and known by barangay officials and women. However, non-reporting of violence is still an issue. This study shows that every community's culture and practices play an important factor in how violence against women was addressed despite what the law says and on the protocol provided by law. This culture also dictates how victim-survivors are treated. Assisting people in need is observed not because it is dictated by law but because it is the right thing to do in such a situation.

The homogeneity of a community somewhat dictates how violence against women is addressed. The barangays with the heterogenic population in terms of culture apply the law when the ones involved are immigrants. This is, however, not the case in instances where the victim-survivor is a native or has Kalinga blood. When this is the case, their elders intervene and influence the victim-survivors decision to settle or file a case (where usually the settles on the first).

Lack of concrete information to show the extent of VAW in the country as many cases of violence against women go unreported due to women victims' "culture of silence" posed a problem. Many of the victims are ashamed to relate their experiences, while others tend to dismiss their ordeal as a result of their lack of faith in the country's justice system caused by frustrations over the lack of results in filing complaints.

\section{CONCLUSIONS}

Women in an intimate relationship experienced one or more modes of violence during or after their relationship duration. However, the frequency and reason for not reporting may vary from one victim-survivor to another. A need to empower victim-survivors that they may not hesitate to report violence committed against them by their intimate partners may be initiated. For this reason, an extension project with the banner program of "Protection of Women against Violence" was initiated and extended to the different barangays starting from two of the barangays where women respondents come.

\section{REFERENCES}

[1] Republic Act 9262 and IRR. (2004). Retrieved March 6, 2015, from Philippine Commission on Women: http://www.pcw.gov.ph/sites/default/files/documents/resource s/ra_9262_

a. and_irr.pdf

[2] RA 9851. (2009, December 11). Retrieved July 15, 2015, from The LawPhil Project: https://www.lawphil.net/statutes/repacts/ra2009/ra_9851_200 9.html

[3] American Addiction Centers. (n.d.). Short and Long Term Mental Effects of Alcohol. Retrieved April 11, 2018, from American Addiction Centers: https://americanaddictioncenters.org/alcoholismtreatment/mental-effects/

[4] Cherry, K. (2017, May 13). What is Diffusion of Responsibility. Retrieved June 17, 2017, from Very Well Mind: https://www.verywellmind.com/what-is-diffusion-ofresponsibility-2795095

[5] Cherry, K. (2018, February 11). Social Exchange Theory. Retrieved April 10, 2018, from verywellmind: https://www.verywellmind.com/what-is-social-exchangetheory-2795882

[6] Crossman, A. (2017, August 11). Feminist Theory in Sociology. Retrieved April 12, 2018, from ThoughtCo.: https://www.thoughtco.com/feminist-theory-3026624

[7] Cycle of Violence. (n.d.). Retrieved April 13, 2018, from Criminal Justice: http://criminaljustice.iresearchnet.com/crime/domestic-violence/cycle-ofviolence/

[8] Fernandes-Alcantara, A. L. (2017, September 13). Family Violence Prevention and Services Act (FVPSA): Background and Funding. Retrieved April 14, 2018, from Family Research Service: https://fas.org/sgp/crs/misc/R42838.pdf

[9] Kantor, J. (2014, September 9). Seeing Abuse, and a Pattern Too Familiar. Retrieved December 13, 2017, from The New York Times: https://www.nytimes.com/2014/09/10/us/seeingabuse-and-a-pattern-too-familiar.html\#story-continues-1

[10] Minnesota Advocates for Human Rights. (2003). Causes and Theories of Domestic Violence. Retrieved April 14, 2018, from http://hrlibrary.umn.edu/svaw/domestic/ training/materials/TheoriesDV.PDF

[11] Republic Act 8353. (n.d.). Retrieved July 18, 2015, from Philippine Commission on Women: http://pcw.gov.ph/law/republic-act-8353

[12] Republic Act 8505. (n.d.). Retrieved July 15, 2015, from Philippine Commission on Women: http://www.pcw.gov.ph/law/republic-act-8505

[13] Republic Act No. 9995. (n.d.). Retrieved July 15, 2015, from The LawPhil Project: https://www.lawphil.net/statutes/repacts/ra2010/ra_9995_201 $0 . h t m l$

[14] Reven, E. (2010, July 08). The Value of Shame ("Hiya") to Filipinos. Retrieved April 11, 2018, from La Solidaridad: http://laonlaan.blogspot.com/2010/07/value-of-shame-hiyato-filipinos.html 
[15] Seigel, L. J. (1998). Criminology Theories, Patterns, and Typologies 6th Edition. In L. J. Seigel, Criminology Theories, Patterns, and Typologies 6th Edition (pp. 102-103). Wadsworth Publishing Company.

[16] Swanson, K. (2014, March 28). Crime Against Women - A Brief History of Laws in the US. Retrieved May 5, 2015, from GetInclusive: https://www.getinclusive.com/blog/crimewomen-brief-history-laws-us/

[17] Taylor, S. (2012, August 30). Why Men Oppress Women, The Psychology of Male Domination. Retrieved April 28, 2018, from Psychology Today: https://www.psychologytoday.com/us/blog/out-thedarkness/201208/why-men-oppress-women 